\title{
Protée
}

\section{" Des stries et du noir " aux rythmes et aux lumières. Une description de sept peintures de Pierre Soulages}

\section{Marie Renoue}

Volume 27, numéro 3, 1999

L’imaginaire de la fin

URI : https://id.erudit.org/iderudit/030576ar

DOI : https://doi.org/10.7202/030576ar

Aller au sommaire du numéro

Éditeur(s)

Département des arts et lettres - Université du Québec à Chicoutimi

ISSN

0300-3523 (imprimé)

1708-2307 (numérique)

Découvrir la revue

Citer cet article

Renoue, M. (1999). « Des stries et du noir » aux rythmes et aux lumières. Une description de sept peintures de Pierre Soulages. Protée, 27(3), 110-118.

https://doi.org/10.7202/030576ar
Résumé de l'article

Adoptant la démarche sémiotique de l'École de Paris et les acquis de la phénoménologie merleau-pontyenne en matière de perception et d'esthétique, cet article propose une lecture paradigmatique et syntagmatique d'une série de sept peintures de Pierre Soulages. Mode d'exposition, sérialité, formes et substances - lumière et rythme - mobiles de l'expression sont analysés, définis face à un sujet culturel présupposé. La mobilité de la saisie perceptive du sujet modalisé par les peintures qu'il regarde nous invite à considérer la perception comme un processus sémantique dynamique et à nous interroger à nouveau sur l'évidence du regard percevant. 


\section{«DES ST RIES ET DU NOIR» AUX RYTH MESET AUX LUMIÈRES UNE DESCRIPTION DE SEPT PEINTURES DE PIERRE SOULAGES}

M ARIE Ren OUE

Choisir comme objets d'analyse des «œuvres d'art», en I'occurrence une série de sept peintures de Pierre Soulages, n'est pas anodin pour une sémiotique de la réception et de la praxis énonciative telle que nous la pratiquons. Le statut sociolectal de ces objets présentés comme «esthétiques» semble assurer leur val eur, les doter d'une prégnance qui en favorise et complexifie l'appréhension et la perception. F uvres prégnantes, elles ont également cet avantage de mettreà mal le spectateur et le commentateur qui setrouvent al ors confrontés à une sorte de carence du sens, de sémantique en creux. Q ue dire du contenu de toiles «noires et striées»? Comment traiter des dimensions visuelle, sémantique et esthétique de ces objets aux titres peu évocateurs?

Cette obscurité signifiante de l'objet semble en fait un manipulateur énonciatif capable d'inciter le sujet à demeurer avec l'objet, à en observer avec précision la forme de l'expression, une forme qui s'avérera changeante, en devenir, car soumise aux mouvements et aux transformations de la substance. 0 ubliant sa préférence passée pour la forme, le sémioticien greimassien severrait donc de nouveau confronté à une description des modulations et de la substance, à une approche du devenir et de l'en deçà de la forme; et ceci d'autant plus que les toiles peintes ne semblent ni noires ni striées, mais queles rythmes et les lumières y travaillent sous I'œil du spectateur attentif et sensible.

\section{M odalités de la visée et de la saisie d'une série de sept peintures}

La description de ces objets, des jeux de lumière et de rythmequ'ils présentent présuppose en effet un regard, celui d'un sujet observateur doté de compétences culturelles qui orientent les parcours interprétatifs auxquels donne lieu I'objet. En deçà de la description apparaîtraient ainsi pour le sémioticien les compétences potentialisées - typifiées et mémorisées - que peut convoquer l'objet; des savoir-faire et savoir-être orientant le mode de visée et le mode de saisie que le sujet réajustera au fur et à mesure de la perception complexe et en devenir de l'objet. Analysant la forme d'objets, noustraiterions ainsi dela relation sujet-objet, d'une interrelation où les deux instances, objective et subjective, s'interdéfiniraient, semodaliseraient réciproquement sur les plans pragmatique ${ }^{1}$ et noologique ${ }^{2}$ compte tenu de val eurs diversement aspectualisées dans une société.

Pour la définition ou du moins pour le balisage de la visée de l'objet, sa catégorie sociolectale joue en effet un certain rôle. Le statut culturel qui lui est assigné linguistiquement ou contextuellement dessineun horizon d'attente, actualise des formes de saisie culturelles, une axiologie et la possibilité d'évaluations que le sujet peut accepter ou non d'assumer. «吕 uvres d'art», les objets qui nous intéressent sont ainsi dotés de valeurs spécifiques qui en font des objets privilégiés du regard, des objets que leur statut et leur forme de tableau «déictisent», rendent présents et saillants à I'attention d'un sujet réceptif. Ce caractère exhibitionniste de la toile peinte, sa valeur intense dans le domaine de la perception en font un manipulateur remarquable. II semble ainsi capable de retenir les pas du visiteur, de plus ou moins I'inciter à se déplacer ou à se poster à une bonne distance, celle, écrit M . M erleau-Ponty, où l'objet demandeà êtrevu 3 . Jouant d'autres effets visuels que ceux de la perspective classique, les toiles de Pierre Soulages laissent le sujet peutêtre plusindécis dans son positionnement; ceci d'autant plus que les peintures présentent une sobriété formelle caractéristique, qu'elles paraissent d'abord donner «peu à voir». Et cette indécision visuelle semble pouvoir rendre le spectateur encore plus libre de ses mouvements, donc plus soumis et peut-être plus attentif aux variations de lumière, de couleur, de forme que provoquent ses déplacements, à une mobilité des apparences qui peut lui indiquer de façon réflexivel'impact de son action sur la perception des objets. 
U neperception qui, diversement modal isée dans le domaine culturel de l'art, peut se faire analytique, comparative ou plus impressive, esthétique, ainsi que l'ont évoqué $M$. M erleau-Ponty 4 et nombre d'écrivains.

Avec le contexte sociolectal et culturel qui dessine un mode de réception des objets, le contexte physique de leur exposition modalise également lespectateur. Leur entourage, I'espace qui les reçoit, le type de leur présentation, de leur accrochage, influencent leur visée, accentuent leur présence ou les gomment en imposant un type de saisie, qui sera plus ou moins parcellaire, frontal ou circulaire, et en jount syntaxiquement d'effet de contraste; le contrasteremplissant alors son rôle d'opérateur de la perception assurant la délimitation, la segmentation et le degré de saillance des objets et des formes. Présentés dans l'atelier blanc et à la lumière diffuse de Pierre Soulages ${ }^{5}$, les tableaux profitaient ainsi d'un contraste coloré maximum. L'intensité de leur présenceétait également accentuée par la disposition en cercle des toiles aux dimensions imposantes $(222 \times 157 \mathrm{~cm})$; cette concentration circulaire comprenait, englobait le spectateur dans un mouvement continu capable de mettre en valeur les continuités ou rupturesformelles entre les différentes œuvres spatialement et visuellement rassemblées. Leur succession favorisait ainsi une lecture syntagmatique que leur pré sentation verbale comme série et leur numérotation cachée sur le châssis invitaient également à entreprendre.

L'ensemble de peintures quenous avons choisies comme objets d'analyse se présente en effet comme une série, un ensemble structuré relativement homogène, mais d'une homogénéité différente de celle des polyptyques réalisés ailleurs par Pierre Soulages. La différence d'abord formelle entre ces deux types de «groupement» n'est pas sans importance pour l'appréhension et la réception des œuvres. Contrairement aux polyptyques dont la coordination - plutôt que la continuité - est réal isée par le cadre ou par le rapprochement, la série joue de discontinuités ${ }^{6}$, de césures en vide qui séparent matériellement les peintures. I ci, l'absence d'un cadre qui isolerait chaque production, en soulignerait les limites intérieures, n'accentue cependant pas la solution de continuité, une solution niée d'ailleurs par la réapparition de motifs linéaires, de jeux de lumière dans les tableaux voisins. En fait ceux-ci peuvent apparaître pour le spectateur qui en suit l'enchaînement commedes traces d'états différents d'une peinture en transformation. Dynamisé par la disposition circulaire et scandée des grandes toiles, le regard de I'observateur syntagmatiserait ainsi ce qui apparaît en suc- cession. Et le rythme visuel formé par la suite de tableaux et de vides spatiaux proposerait entre chaque forme peinte l'espace d'un repos pour le regard, ou plutôt d'un repos qui, modalisé par l'apparition de toiles en résonance, deviendrait ensuite attente de transformation et virtualisation d'étapes intermédiaires.

Face au polyptyque qui oppose, souligne davantage par la juxtaposition les ruptures formelles, la série proposerait ainsi presque paradoxalement par levide un espace de conciliation possible, decontinuitévirtuelle; I'opposition formelle serait ainsi adoucie, amoindrie et le rythme perceptif de l'ensemble se ferait moins brutal, moins heurté. Scandé et circulaire, cemouvement desyeux n'est néanmoins pas infini; il est orienté entre des limites, le début et la fin de la série invisibles sur le châssis, mais formellement et spatialement indiqués par une estrade de bois sur laquelle est placée la dernièretoile, la plus dépouillée, alors queles autrestableaux sont posés à même le sol, contre le mur ou les piliers.

Cette présentation des tableaux dressés contre les parois accentuel'impression de massivité, de matérial itédes œuvres.
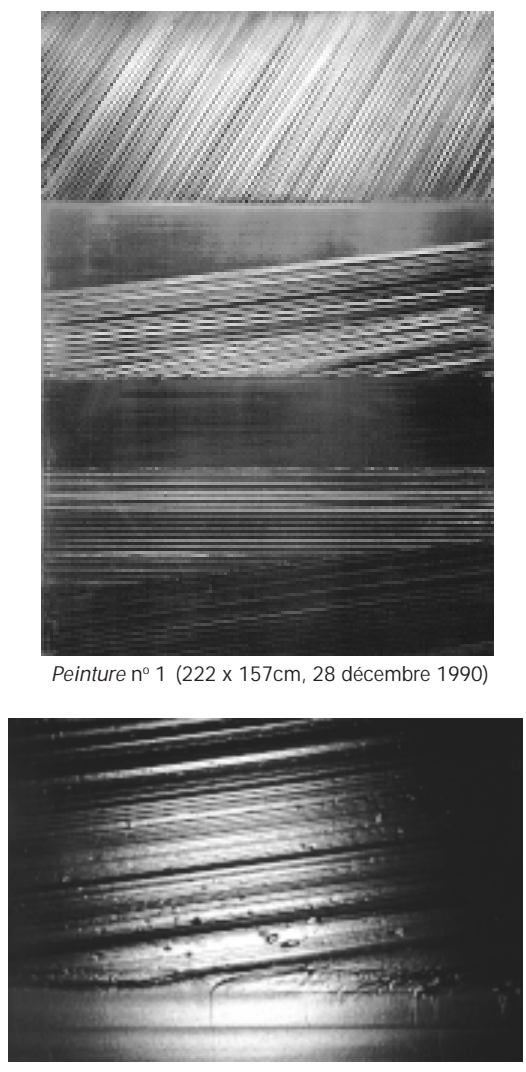

Détail de Peinture $n^{\circ} 1$ 
Prégnantes par leur position, imposantes par leurs dimensions, elles s'affirment d'abord comme des objets matériels, ainsi que l'indique leur titre qui laconiquement mentionne la technique: Peinture, le format: $222 \times 157 \mathrm{~cm}$ et la date variable de la réalisation 7 . Ce que nous donnerait à voir chacune de ces œuvres serait donc de la peinture étalée sur un espace mesuré. Seule la date renvoie à autrechose, donne lieu àun débrayage sur le temps de la production, un ancrage temporel qui permet de rapporter les objets à un faire, à la chronologie de la production de l'artiste. Un artiste qui, comme nombre de ses contemporains, ne signe pas ses œuvres; l'attribution sera dans les musées autrement assurée par l'étiquetage ou plus directement par le procès d'identification que réalisent les spectateurs actifs, invités de fait à convoquer des marques identitaires potentialisées, à «reconnaître» des objets, puisuneidentité derrière des variations grâce à un examen stylistique, figural del'objet, c'est-à-dire, pour lesémioticien, grâceà la construction del'objet deval eur en tant quetel 8 .

Sans signature, dotées de titres sans histoire, les œuvres inciteraient ainsi le sujet d'abord à focaliser son attention sur la peinture, sur sa matièreunique et noirequi, recouvrant le support, génère des formes eidétiques et lumineuses.

\section{T extures: formes et matière}

O ubliant la prégnance des peintures disposées en cercle, celle des formes linéaires et des aplats qui se répondent, le spectateur peut se déplacer petit à petit, s'approcher des œuvres pour percevoir davantage la matière picturale, la texture de l'enduit qui recouvre toute la surface de la toile. La texture de chaque tableau considérée de manière plus statique apparaît régulière, articulée autour d'une opposition entre la surface lisse d'aplats de différentes dimensions et des ensembles de stries, de rainures parallèles qui traversent horizontalement ou en obliques montantes ${ }^{9}$ des espaces bien délimités. II n'y a pas ici de foisonnement désordonné, de formes contradictoires qui brouilleraient ou intercepteraient le regard. Le sujet peut suivre dans un mouvement continu et régulier les rainures profondes et larges qui creusent la masse sombre de l'enduit. D 'aspect général régulier, ces rainures présentent cependant quel ques petites variations de profondeur, de largeur qui donnent lieu à des oppositions plus ou moins accentuées deluminosité, des oppositions qui mettent en valeur des lignes au détriment de l'ensemble isomorphe du champ. Plus près, le spectateur peut être plus attentif et arrêté par ces tranchées qui creusent la masse picturale, par le relief aigu de leurs bordures et par le débordement de l'enduit un peu épais qui longent parfois les rainures ou encore par le doublement parcellaire dela forme linéaire.

Sousun point devueplus rapproché, la régularitéformelle s'altère davantage encore; le matériau présente parfois, à l'intérieur des rainures, sur leurs bordures et dans les aplats «lisses», de fines et profondes rayures, des trous épars irréguliers, des bulles, des renflements ou même des «désé paississements», sortes d'effeuillage d'une légère pellicule d'enduit. Ailleurs, la surface de l'enduit ondule le long des stries, de légères vagues traversent les aplats, créant ainsi de fines ou larges modulations de luminosité qui en soulignent les formes. La régularitéformelleest donc affaire de point de vue, de distance et defocal isation. $\mathrm{N}$ iée parfois par les traces doublées et diversement appuyées qui creusent les stries dans la matière, cette régularité relative perçue d'emblée semble en tout cas l'affaire de la forme donnée, tandis que l'irrégularité, plus invisible, parait tenir davantage au matériau, à son expression propre, à sa résistance et à sa densité, facteurs çà et là de bulles et de débordements, de repliements de la matière travaillée, étirée. Entre régularité et irrégularité, la texture apparaîtrait ainsi comme la cristallisation d'états de la matière, une matière travaillée et plus ou moins réactiveà la tension que lui impose la forme. Au-delà de la description, il y aurait ainsi I'amorce possible d'une narrativisation, I'histoire de transformations réciproques, celle d'une forme (ou déformation) imposée à un matériau dense, compact et résistant qui donnerait en retour son aspect, sa stabilité à la forme, mais aussi ses petites imperfections.

\section{La texture, la couleur et la luminosité: variations spatiales et temporelles}

D ans ces œuvres dePierreSoulages, la matière, la texture génère des formes eidétiques, colorées et lumineuses. La congruence de ces différentes qual ités retenues généralement lors de description des objets est ici évidente. Car le creusement de l'enduit dessine des lignes lumineuses qui seront plus ou moins soulignées dans le temps en fonction de l'orientation de l'enduit par rapport aux sources de lumière qui éclairent et illuminent les peintures. La lumière se réfléchit, se transforme dans les creux, sur le relief de la matière noire qui recouvre la toile. Et cette «couleur» noire est sans aucun doutel'un des éléments les plus prégnants de ces œuvres, une marque identitaire au point que certains commentateurs n'ont pas hésité à nommer Pierre Soulages 
«le peintre du noir». Le noir qui apparait d'abord sur toute la surface de la toile semble intense, saturé et satiné, légèrement lumineux et variant d'intensité avec l'éclairage ambiant. Cette relation paradoxale entre le noir et le lumineux, généralement dissociés dans notre culture - au plan de l'expression où le noir est confondu avec l'ombreux et, defait, au plan du contenu à cause des valeurs axiologiques négatives associées à l'ombre et au noir - , apparaît en raison de son apparente contradiction relativement saillante. II s'agirait en quel que sorte pour le spectateur de réviser le motif deleur dissociation ou disjonction réciproquepour percevoir les modalités de leur relation.

L'aspect satinédel'enduit et sa coul eur sombre pourraient ainsi semodaliser réciproquement et différemment. $D$ 'après J.G. Sjölin 10 qui note la capacité d'une surface brillante à soutenir les val eurs du noir quand les surfaces ternes rendent les mêmes valeurs grises, brillance continue, le satiné participerait à l'intensité du noir. Ajoutons par ailleurs que cette intensification de la saturation de la couleur par la brillance semble également corrélée à la saillance perceptive du lumineux; la brillanceet ses gradations mettent en valeur les objets, les démarquent et disjoignent d'un fond, favorisent par la réflexion intense et rapide de la lumière un effet de surface ${ }^{11}$ plutôt que de matière, de profondeur, lesimposent ainsi directement et rapidement au regard comme des surfaces brillantes et colorées. M ais la relation entre la brillance et la couleur semble parfois plus polémique; en favorisant la réflexion de la lumière, une brillance trop intense paraît en effet pouvoir nuire à l'aspect coloré des objets. Satinées, les peintures de Pierre Soulages peuvent à cet égard apparaître d'abord comme un modus vivendi. Elles présentent néanmoins au spectateur des variations de luminosité et d'apparence colorée suivant la texture de l'enduit.

Plus uniformes, les aplats semblent en effet présenter un noir et une luminosité moins intenses que ceux des stries où ombres et lumières s'opposent plus brutalement entre l'intérieur sombre des rainures et les arêtes lumineuses des bordures. Les stries jouent du contraste, del'opposition entre intensités d'un noir profond, ombreux et d'un noir lumineux pour souligner les formes linéaires donnéesà la matière, quand la surface plane de l'enduit semble plus sage, moins intensive avec seulement parfois des oppositions moindres et en continu de luminosité. Le contraste brutal formé par les stries semble ainsi à mêmed'intensifier les valeurs perceptives deluminosité, mais aussi de couleur. C ependant, pour le regard attentif davantagefocalisésur les stries, la corrélation inverse entre inten- sités importantes de luminosité et de couleur évoquée peu auparavant est manifeste; plus la ligne dessinée par la rainure est profonde et sombre, plus elleparaît noire, à l'inverse le lumineux relief semble perdre de son intensité colorée. Accentuées par l'effet de contraste, luminosité réfléchie et couleur varient ainsi concurremment d'intensitéet de force perceptive. Avec le déplacement du sujet, le spectacle lumineux change; les taches lumineuses se déplacent sur la surface éclairée qui devient alors plus ombreuse ou lumineusesuivant l'angle devueprivilégié. Sousleregard du spectateur qui sedéplace, qui s'approche pour voir davantage les ombres portées et les lumières, qui longe les surfaces enduites pour saisir les variations lumineuses, les peinturesapparaissent ainsi mobileset changeantes.

Variableet lumineuse, la couleur del'enduit semble en fait peu noire ou, du moins, noire peu de temps. La stabilité perceptive évoquée par les physiologistes et les phénoménologues comme M. M erleau-Ponty dépend du regard; le noir des tableaux perçu d'emblée semble ici plutôt interrogé, et ce dans ses relations avec la luminosité, c'est-à-dire avec la réflexion de la lumière extérieure. Cible directe de la lumière qu'aucun cadre $^{12}$ nevient contenir, la peinture varie en effet également en fonction de l'éclairage ambiant. Satiné, l'enduit noir en modalise néanmoins la réflexion. La lumière réfléchie sembleen effet contenue; même dans ses intensités extrêmes, elle apparaît moins intense que l'éclairage qui la donneà voir. Atténuante, amenuisante, la peinture semble transformer la lumièreen devenant, plutôt qu'unesimplesurface réfléchissante, une seconde source ${ }^{13}$ d'une luminosité qui, plus retenue, plus atone, sourd de la toile. Lumineux, variant avec l'éclairage, son intensitéet même sa teinte, «l'enduit noir» ascétique des peintures devient un support où luminosités plus ou moins intenses et chaudes varient et font varier la couleur. Ce serait donc à une expérience visuellefaisant fi de la stabilité perceptivedu noir queserait conviéle spectateur des peintures. II pourrait tenter de percevoir,

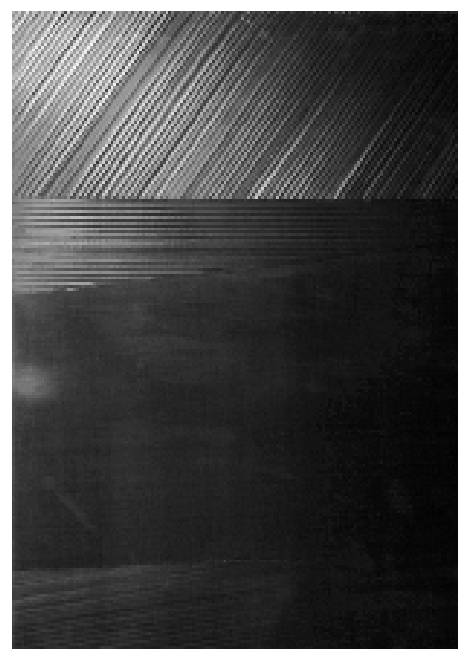

Peinture $\mathrm{n}^{\circ} 2$

(222 x 157cm, 30 décembre 1990)

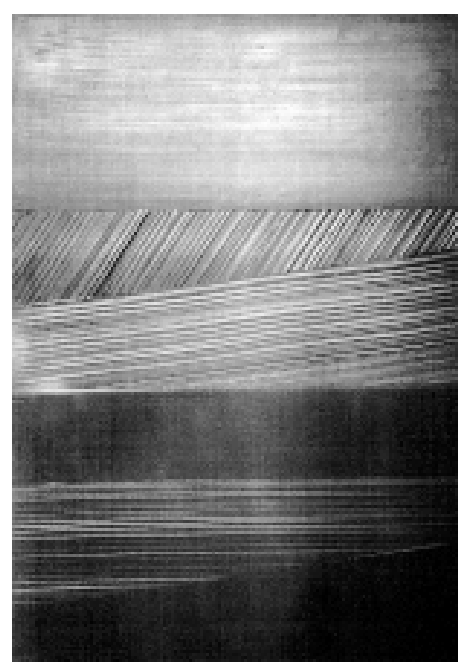

Peinture $\mathrm{n}^{\circ} 3$

(222 x 157cm, 5 janvier 1991)

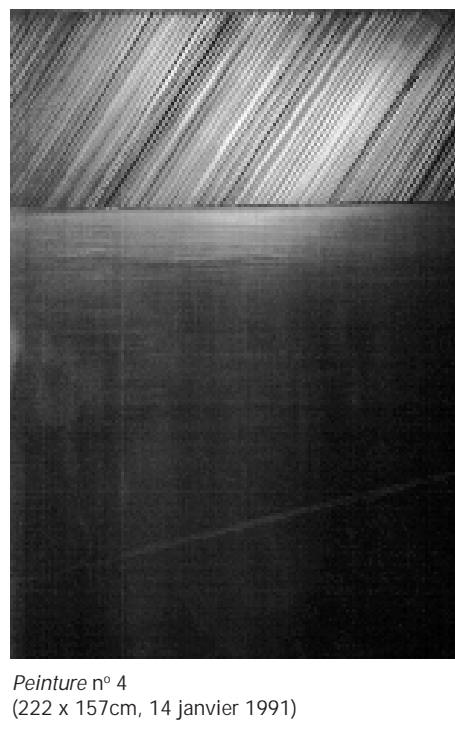



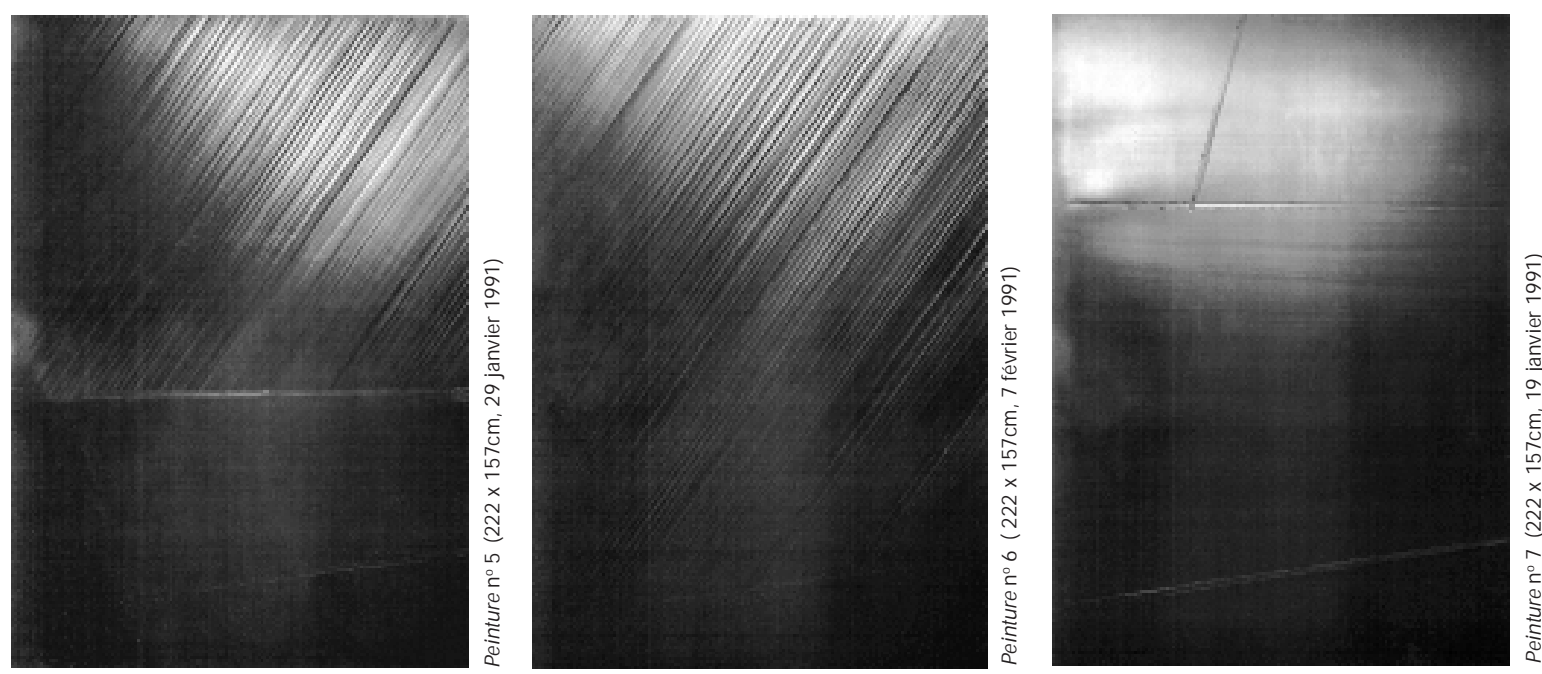

derrière leur apparence austère, la richesse de variations que peut receler un noir satinés'exprimant non commenégation de la lumière et de la teinte, mais comme second générateur potentiel de luminosité et de modulations colorées.

D épendant de la lumière extérieure, les peintures changent évidemment d'aspect lorsque l'éclairage est moins intense. Alors, leur aspect satiné s'estompe, leur luminosité faiblit et les tableaux sombres semblent gagner en intensité de couleur, en densité de présence, de matérialité ce qu'ils ont perdu en intensité et en oppositions lumineuses. T out se passe commesi matière et lumièreétaient en concurrence, la densité et massivité de la présence statique de l'une s'opposant à la vibration changeante, mobileet plusallégeante de l'autre. La présence massive des toiles perçues d'emblée que nous avons évoquée auparavant serait ainsi variable. D 'abord prégnante, puis moins intenselorsquele spectateur se familiarise avec les objets, focalise son attention sur un aspect, texture, couleur ou luminosité, cette présence redeviendrait plus imposante et plus statique avec la baisse de l'éclairage et des oppositions lumineuses et eidétiques présentées par les toiles. D ynamisme visuel et perceptif dans un cas, rétention dans I'autre cas; I'intensité lumineuse et celle des modes de densité de présence matérielle varieraient de manière inverse et modaliseraient diversement la perception du sujet en jouant d'effets de contraste, de surface et de profondeur.

\section{Aplats, stries et lignes: de la concentration et du rythme}

$D$ ans les conditions de visibilité habituelles des œuvres d'art, à savoir un éclairage qui en favorise la saisie, la pré gnance, les peintures de Pierre Soulages présentent, avonsnous dit, une grandeopposition eidétiqueentre stries, lignes et aplats. Formes lisses, continues, peu propices aux contrastes, les aplats paraissent plus atones, moins saillants que les formes striées sombres et lumineuses qui traversent les peintures. Fortement gravées dans la masse, disposées parallèlement les unes aux autres, les stries semblent dotées d'une certaine force énonciative; elles paraissent en effet pouvoir orienter le regard du spectateur, en retenir et dynamiser le trajet le long des axes rectilignes qu'elles dessinent. I solées, les lignes semblent perdre de cette force directrice mal gréun tracé parfois doublé, appuyé comme pour lui donner plus d'intensité. D e par leur relative unicité, celles-ci requièrent en fait attention, concentration pour suivre leur trajectoire dynamique, quand les stries parallèles pouvaient récupérer sans dommage un regard divergeant et que l'aplat invitait à l'expansion. Entrecesaplatsqui proposent au regard l'espace d'un repos, les lignes isolées et les zones striées dotées d'intensité et de concentration propres, les peintures semblent traversées d'un rythme perceptif irrégulier, composé des silences de la forme unie qui s'épanche et du dynamisme plus ou moins rapide et concentré provoqué par les lignes suivies du regard.

Est-ce à dire que les aplats formeraient un fond pour les motifs linéaires? En fait, la chose nous semble un peu plus complexe. La perception, la sensation de profondeur par exemple n'est pas vraiment associée à l'aplat commeil en va généralement $d u$ fond; les stries proposent à côté du lumineux relief une sombre profondeur - réelle et perceptive - bien supérieure. Par ailleurs, la saillance de ces zones unies ou striées semble dépendre en partie de leur expansion, deleur importancequantitativedansla toile, ou parfoismême des variations présentées par des peintures voisines. Ainsi, la seconde toile de la série semble-t-elle presquefaire remonter à la surface l'aplat par l'importance spatiale et situationnelle qu'ellelui donne; un repospour le regard qui vient dequitter la première peinture traversée par les champs de stries qui s'opposent formellement et se heurtent par leur rythme différent. La quatrième peinture semble davantage poser la question de cette interprétation visuelle. L'importance de 
I'aplat, sa remontée perceptive est un peu contredite par une ligne unique qui le traverse comme un motif sur un fond (ou qui sépare l'aplat en deux), tandis que les deux toiles suivantes, en augmentant l'expansion des stries, semblent presque lui assigner un rôle de fond sur lequel glisserait petit à petit un champ plus intense et contrasté. En fait, plutôt que cette opposition fixée et souvent sémiotiquement pertinente entre fond et figure, il nous semble préférable d'évoquer des variations rythmiques, des mises en valeur contextuelles de tel ou tel champ qui le dotent de valeurs de profondeur ou d'intensité visuelle particulières.

Contrairement aux aplats formellement différenciés par leurs seules dimension et position dans les toiles, les zones striées et les lignes présentent également des formes diffé rentes, des orientations variées qui influencent diversement la perception. H orizontales, elles peuvent égal ement adopter des angles d'environ $170^{\circ}$ ou $125^{\circ}$. Des formes qui évidemment setransforment, se lèvent davantage ou se baissent avec le déplacement latéral du sujet et de son angle de vue. Pour un spectateur immobile, l'axe dessiné semble précis, I'orientation est assurée le long de ces axes rectilignes et dynamiques. Parfoiscependant les limites des stries montrent des courbures, de légères inflexions vers la gauche, là où le regard occidental les voit apparaitre, et un amenuisement vers la droite avant leur disparition; ailleurs c'est à gauche ou vers le haut que les lignes apparaîtront estompées, moins appuyées. La manifestation de la forme linéaire semble ainsi remettreparfois en question une régularitéformelle sansfaille, ou plutôt celle-ci est diversement aspectualisée le long de son trajet. Apparition et disparition de la forme sont marquées par un mouvement courbe qui se fait droit ou par l'effacement, la disparition dela profondeur: c'est à l'intérieur du tableau plutôt qu'à proximité de ses limites, de son cadre que les formes plus fortement dirigées et gravées s'expriment avec le plus d'intensité, de force directrice et dynamique.

D 'autres phénomènes d'estompage apparaissent ailleurs, à la frontière entre deux champs différents, là où une forme disparait pour laisser place à une autre. En fait, outre ce passage en continu d'une forme à l'autre par l'intermédiaire d'une disparition graduelle et imprécise, cette délimitation des zones peut se faire de façon plus brutale, par la coupure, l'arrêt de stries par l'apparition des stries du champ voisin, voire par l'apparition plusincisived'unestrie de démarcation horizontale ou légèrement oblique (environ $170^{\circ}$ ). Parfois, des chevauchements, des superpositions, brouillent un peu cepassage entre champs voisins. Lazone de démarcation, de limitation, sembledonc un espacevariable, propreà latension et à la narrativisation, un espace rythmique discontinu aussi, où des dynamismes différents peuvent sejuxtaposer, où une rupture, une frontièrelinéaire, peut créer un heurt, un creux parfois outrepassé. Les lignes isolées qui apparaissent dans les dernières peintures, principalement dans la septième, posent d'autres problèmes perceptifs et interprétatifs. Épaisses, profondes, voire même doublées par endroit, elles peuvent apparaître plus comme des motifs saillants, directifs et dynamiques traversant les aplats que comme des lignes de démarcation ou même de jointure entre deux zones identiques. L'interprétation visuelle que nous privilégions, en raison d'unecontinuitéformelle entreles premièreet dernière peintures (nous y reviendrons), laisse évidemment plus de place au dynamisme linéaire et du coup néglige un peu une lecture de rapport demasse entre aplats de différentesformes et dimensions.

$O$ rientant, concentrant le regard, aplats, stries et lignes génèrent des dynamismes perceptifs différents, certes. $M$ ais qu'en est-il du rythmedechaquepeinture? Q uelleimpression rythmique recèlerait une œuvre dans sa gl obal ité, lorsque le sujet changeant de point de vue oublierait la focalisation, le méréologique et le rapproché pour une vue d'ensemble? II semblerait queles différents champsou éléments rythmiques se régulent ou, dans une conception plus polémique, entretiennent un rapport de force où la quantité entrerait en jeu. Ainsi, la seconde peinture nous apparaît-elle globalement relativement calme, mal gré ses zones striées; étendu, l'aplat qui la traverse marque la saisie de son atonie mise à mal cependant par la ligne oblique qui lui sert de démarcation. Le même calme relatif peut apparaitre dans la quatrième peinture où une ligne oblique vient à nouveau dynamiser un peu le grand champ lissequi la reçoit. C onsidérée dans sa globalité, la première peintureaux variations multiples nous apparait au contraire marquée par la scansion, la disparition ou la discontinuité de plages identiques.

Plus attentifs, nous pouvons peut-être être sensibles aux valeurs dynamisantes différentes que possèdent les diverses formes de stries. Le dynamisme de l'obliquité a été maintes fois souligné par les artistes. $D$ ans le contexte de cette série de peintures, à moins d'être reprise à la même hauteur dans une toile voisine, I'horizontale semble en effet relativement peu dynamiquepour le regard. Leslignes montantes, toujours situées dans la partie supérieure des tableaux, voient leur dynamisme enfreint, retenu par la coupure ou la fin de leur champ; dans l'avant-dernier tableau, leur progressive ex- 
pansion assurera le dynamisme contenu ailleurs. Pouvant traverser entièrement la peinture, I'oblique d'environ $170^{\circ}$ nous semble au contraire relativement dynamique. Elle apparaît ainsi, stries ou lignes, dans les différentes peintures, plus précisément dans la partie inférieure. Car les formes semblent plus ou moins localisées et ce de façon inégale. D ès les trois premières toiles, I'aplat semble ainsi pouvoir occuper toutes les places. L'horizontale paraît un moyen terme, celui du pas tout en haut, du pas tout en bas, tandis que les obliques montantes sont situées en haut ou presque et les obliques de $170^{\circ}$ lesont dans ou vers le bas. Si la relation posée entreforme, mode d'expansion et dynamismea raison d'être, les sept peintures que nous étudions proposeraient au spectateur non pasun dynamisme des hauteurs, une sorte d'appel à la transcendance, mais un dynamisme du bas, plus maîtrisé peut-être par ce qui le surmonte. Par ailleurs, les lignes ou stries de $170^{\circ}$ situées vers lebas présentent l'avantage non négligeable de diriger le regard du spectateur non pas vers un ailleurs situé en haut, mais vers la droite, c'est-à-dire vers les autres peintures de la série.

\section{La série de sept ${ }^{14}$ tableaux: tendances à la simplification et au silence}

La série de sept tableaux est assimilable à un paradigme où des motifs établis, ici peu nombreux - aplat, lignes parallèles ou uniques orientées suivant trois voire quatre axes -, seraient localisés dans des champs aux dimensions variables et dans les contextes configurationnels que nous venons de présenter. Au-delà, il s'agirait de considérer les effets rythmiques, perceptifs provoqués par la présence ou l'absence d'uneforme, ou par la variation de l'importance quantitative qui lui est accordée. Cette variation des formes, de leur présence, de leur quantité peut donner lieu, nous y avons déà eu recours, à une lecture syntagmatique où l'on jugerait alors de l'expansion des formes, de leur apparition ou disparition dans deux peintures contiguës. Syntagmatisant notre perception de la série, nous pourrions ainsi remarquer tout d'abord une sorte de scission ou du moinsun tournant entre les trois premières peintures et les suivantes. Plus précisément, la quatrième peinture jouerait en quelque sorte un rôle de pivot entre deux tendances, deux types de transformation que nous définirons successivement.

D e la premièreà la troisième peinture, c'est certainement I'impression d'une complexité relative qui domine, non une complexité qui serait due à la variété plus importante des motifs - toutes les toiles excepté la sixième présentent avec des aplats les trois axes sous forme de stries ou de lignes -, maislenombre dechamps délimités y est supérieur et surtout, en l'absence de lignes isolées, ce sont, avec les aplats, des zones de stries qui occupent tout l'espace, le dotent de la densiténumérique et de présenceévoquée auparavant. $D$ ans la première peinture apparaissent ainsi six zones, dont deux discontinues ou répétées, d'aplat et d'obliques. La scansion de l'espace entier qui en résulte est évidemment heurtée et rapide, et ce d'autant plus que les aplats sont comme comprimés par le dynamisme des stries voisines.

La peinture suivante paraît beaucoup plus calme; l'aplat s'est élargi au centre de la composition, entre les obliques et les horizontales qui, moins développées, perdent un peu de leur dynamisme. M al gréune réduction du nombre dezones, leur forme, la configuration générale de la toile évoque pour partie celle dela premièrepeinture qui apparaît en fait comme unesorte de guide, de canevas de décomposition potentielle des toiles en zones aux formes globales normalisées. La même zone destriesmontantes occupelehaut dela peinture, ensuite le même zonage est repristandis que le bas a été modifié par I'apparition uniqueà cet endroit d'uneligne de démarcation horizontal eet non oblique. M ais, par un chassé-croiséformel, la troisième peinture oublie la tendance à la simplification annoncée et reprend avec six zones le même schéma compositionnel que la première toile. N éanmoins, de sa voisine, cette troisième toile semble conserver un peu du rythme ralenti ; l'aplat y occupe en effet de plus en plus de place, non plus sous la forme de l'expansion antérieure, mais sous forme fractionnée dans les trois zones placées en haut, en bas et vers le centre.

Poursuivant le schéma rythmique croisé qui associe par leur configuration les peintures numéros un et trois, la quatrième reprend la tendance à la simplification annoncée par la deuxième décomposée en quatre champs. Les zones délimitées, toujours conformément au modèle donné d'emblée, sont maintenant au nombre de deux; les obliques montantes ont retrouvé leur site et l'aplat s'est élargi considérablement sur le reste dela toile. U nenouvelleforme, celle de la ligne isolée, apparaît; simplifiée, unifiée, elle reprend la forme oblique des stries ou de la ligne de démarcation qui se trouve un peu plus bas dans les autres peintures. N ouvelle, car isolée et mise en valeur sur l'aplat, cette forme fortement appuyée semble donc découler logiquement des autres lignes, tout comme cette nouvelle peinture parait issue des autres, et semble poursuivre une tendance à la simplification, à l'importance donnée à l'aplat 
et à son rythme particulier néanmoins dynamisé un peu ici par la nouvelle venue qui le traverse. Rattachée au début de la série, cette quatrième peinture apparaît pourtant tiraillée vers une autre tendance à laquelle elle donne prise, une suite de la série qui, reprenant des motifs, des formes de champs, n'est pourtant pas totalement disjointe des premières peintures. II convient néanmoins de noter que cette continuité semble commegommée par lenouveau mouvement qui relie fortement les peintures quatre, cinq et six en une succession parfaite.

En effet, les deux peintures suivantes reprennent de la quatrième toile les stries montantes, les aplat disposés en haut et en bas, avec une tendance contraire à l'expansion. D ela quatrièmeà la sixième peinture, nous assistons en trois étapes à l'extension de la zone striée qui, pratiquement doublée puis triplée, semble occuper petit à petit toutes les zones et glisser ou recouvrir l'espace nu de l'aplat. Cette expansion progressive et nouvelle d'un champ d'obliques auparavant relativement limitésembleindiquer unenouvelle tendance et une nouvelle dynamique; dans un mouvement potentiellement continu d'expansion vers le bas, les champs de stries montantes semblent tendre à envahir tout l'espace faceàl'aplat qui diminued'autant. D ansla sixièmepeinture, la ligne de démarcation horizontale a disparu, remplacée à cet endroit par l'oblique encore un peu épaisse qui contient les stries montantes dans un non-bas. D isparition de l'horizontale et disparition progressive annoncée de l'aplat semblent indiquer au spectateur quela dernière peinture verra la disparition des motifs autres que ces stries montantes, des stries dynamisées par leur expansion progressive dans la peinture. En fait, cette attente créé par la suite progressive est non aboutie; les formes actualisées par la suite de ces trois peintures ne seront pas réalisées, mais directement potentialisées, assumées comme manquantes.

La dernièrepeinturepeut donc apparaitred'abord comme une rupture, un décept: au lieu degrandes et hautes obliques dynamisant l'espace, c'est l'aplat qui apparaît omniprésent. A près la progression des obliques, cet aplat peut apparaître encore plus atone, plus proche du vide, il marque une absence, celle du dynamisme et de l'orientation attendus et son atonie, sa relativenuditéen sont comme mises en valeur par effet de contraste paradigmatique. Le décept assumé, le spectateur peut être plus sensible aux lignes qui traversent I'aplat et le dynamisent à peine. M otifs simplifiés, les lignes évoquent en fait les axes antérieurs, dessinés dès la première peinture par les stries obliques du bas, par la ligne de démarcation horizontale qui limite le champ supérieur et peut-être par les stries montantes du haut, dont l'orientation ascendante serait ici augmentée comme pour marquer une rupture avec les stries envahissantes et dynamisées des peintures antérieures et comme pour «étirer vers le haut», contredire le mouvement de l'horizontale contiguë et ainsi stabiliser davantage la peinture. Tout en affirmant sa différence, la septième peinture semble donc reprendre les motifs antérieurs, l'expansion del'aplat et deson atoniearrêtée dans l'espace des peintures cinq et six, qui proposent une autre démarche dynamique vers la simplification des motifs. La fin de la série rejoint donc le début, mais en substituant à la complexité, à la force énonciative, directrice et dynamique des stries la forme plus subtile, plus simple et silencieuse des lignes et de l'aplat.

\section{N OTES}

1. Cettemanipulation pragmatiqueexercéepar lesobjetset lesœuvresd'art apparait évidente avec les installations «contemporaines» qui, comme celle de $D$ ali dans son musée de Figueras, orientent les spectateurs sur des escaliers, des estrades, et imposent au regard une orientation donnée par la lunette qui seule permet de voir le spectacle créé pour le public. M. Schulz a proposé en 1995 une analyse de l'un de ces dispositifs réalisé par Sol Le W itt. II convient également denoter dansledomainepictural lerôlecoercitif joué par la perspective classique qui impose au regard un axe, un point de mire. Cettefonction manipulatrice est plus explicite avec les anamorphoses qui invitent le spectateur à se doter d'instruments ou à se placer à un point précis de l'espace pour découvrir ce que l'anamorphose affirme cacher, voiler. T el est le cas des Ambassadeurs d'H olbein aux pieds desquels s'élève une forme étrange, une tête de mort invisible de face, mais dont l'étrangeté (lesecret exhibé) éveillela curiositédu spectateur qui sedéplacelatéralement pour percevoir et identifier laforme, et du mêmecoup oublier et neplusvoir les ambassadeurs peints déformés sous ce nouvel angle de vue. 0 bstruction et présentation changent donc concomitamment d'objets; il faut que l'un soit caché pour quel'autre se montre. Et au-delà des jeux perceptifs, c'est le domaine dela véridiction qui est convoqué, une valeur secrète (cequi est et neparaît pas) qui, lorsqu'ellesedévoile, cacheles motifsles plusdirectement apparents et mène finalement au sentiment de l'illusoire (ce qui n'est pas paraît) et du doute, ainsi que l'ont noté les nombreux commentateurs du tableau.

2. Letermenoologiqueprésentel'avantagederelier cognitif et pathémique, laraison et lesensibleartificiellement dissociés. N ouslesconsidéronscomme des «pôles» d'uneactivitéderéception, despôlesqui peuvent apparaîtreplus ou moins intensifiés dans les saisies culturellement dessinées et toujours 
complexes del'esthète- où lepathémiqueprévaudrait - et del'analyste- où le cognitif occuperait la première place.

3. D ans Phénoménologie de la perception, M. M erleau-Ponty écrit: Pour chaqueobjet commepour chaquetableau dansunegaleriedepeinture, il ya une distance optimale d'où il demande à être vu, une orientation sous laquelle il donne davantage de lui-même: en deçà et au-delà nous n'avons qu'une perception confusepar excèsou par défaut... (1945 : 348). La corrélation entre la position du sujet, son point de vue, et la perception de l'objet a été exploitée en art, ainsi que nous l'avons évoqué au sujet des anamorphoses, maisil semble nécessaire de nuancer la généralisation faite quant à la valeur d'un point de vue statique, et ce en raison de la qualité de notre objet et de la libertérelatived'un sujet modernehabituéà regarder certesfrontalement, maisaussi différemment lesœuvres d'art, neserait-cequepour percevoir des détails sou lerelief dela matière. Pour uneétudedeces variationsperceptives, sémantiqueset passionnellesqui affectent lesspectateursmobiles, cf. Renoue (à paraitre)

4. M . M erleau-Ponty traitedecesdiversesformes desaisieperceptivesdans L'CE il et l'Esprit, mais aussi dans Phénoménologie de la perception où il écrit: Selon que je fixe un objet ou que je laisse mes yeux diverger, ou encore que je m'abandonne tout entier à l'événement, la même couleur m'apparaît couleur superficielle ( 0 berfläschenfarbe), - elle est en un lieu défini de l'espace, elle s'étend sur un objet, - ou bien elle devient couleur atmosphérique (Raumfarbe) et diffuse tout autour de l'objet; ou bien je la sens dans mon oal comme une vibration de mon regard; ou enfin elle communique à tout mon corps une manièred'être, ellemeremplit et neméritepluslenom de couleur (1945: 262). Pour une étude de ces modalités de la perception, cf. M. Renoue, 1996.

5. Contrairement aux visiteurs des galeries ou des musées français et étrangers où ces tableaux ont été plusieurs fois exposés, nous avons pu les observer et les photographier dans l'atelier del'artiste, Pierre Soulages, que nous remercions vivement pour sa disponibilité

6. Discontinuité n'est pas discrétion. Alors que celleci marque l'identité d'un objet, délimité et distingué des autres, qu'elle se rapproche de la disjonction, la discontinuité indiquerait comme en linguistique une interruption dans une entité (non-conjonction) ou comme ici la reprise partielle de motifs (non-disjonction).

7. Soit pour les sept peintures suivant l'ordre de leur numérotation, les dates: 28 décembre 1990, 30 décembre1990, 5 janvier 1991, 14 janvier 1991, 29 janvier 1991, 7 février 1991 et pour la septième 19 février 1991.

8. Sur l'identification en art, cf. Caliandro, 1997.

9. L'orientation montante ou descendante des lignes est évidemment déterminée par le sens de lecture adopté par le sujet. D ans la société occidentale, les spectateurs privilégiant une direction de la gauche vers la droite, nous considérons comme montantes les lignes (rectilignes) dont les extrémités apparaîtront plus basses à gauche qu'à droite.

10. D ans J.-G. Sjölin, 1996: 46.

11. Pour une étude des corrélations entre brillance - matité et effets de profondeur perçue (une profondeur évidemment différente de celle «objective» du relief de la texture évoquée auparavant) -, cf. Renoue (à paraître). Cette opposition entre types de profondeur, une profondeur vue et perçue, apparaît également dans J.-G. Sjölin, 1996: 43.

12. Sur le cadre et sa fonction d'équilibrage de la lumière extérieure, cf. $A$. Beyaert-G eslin, 1997.

13. Pour une étude des modalités de la réflexion de la lumière, cf. l'étude de L'Éloge de l'ombre de T anizaki faite par J. Fontanille (1993) et notre étude doctorale (à paraître).

14. C ertains lecteurs seront certainement sensibles à la valeur culturelle et symboliquedu nombresept. Peu enclineàtraiter desymbolismeet soucieuse d'éviter des digressions et des interprétations trop hasardeuses, nous ne désirons pas développer ici ce point, seulement évoquer l'expansion sémantiqueque peuvent entraîner la prise en compte et l'exploitation dece chiffre particulièrement récurrent dans le domaine du religieux.

\section{RÉFÉREN CES BIBLIO GRAPH IQUES}

BASTIDE, F. [1987]: «Le traitement de la matière», Actes Sémiotiques D ocuments IX, 89, Paris, EH ESS, Klincksieck.

BEYAERT-GESLIN, A. [1997]: «Le cadre et la lumière: quelques éclaircissements», Visio 2, 2, 75-87.

CALIANDRO, St. [1997]: «L'identification en art: le procès cognitif de I'attribution », Visio 2, 2, 59-73.

Co URTÉS, ] . [1992] : «D u signifiéau signifiant », N ouveauxActesSémi otiques 21-22, Limoges, PULIM ;

[1991] : Analyse sémiotique du discours, Paris, $\mathrm{H}$ achette.

FLOCH , ..-M . [1985] : Petites mythologies del'œil et del'esprit, Paris, H adèsBenjamin.

FONTANILLE, J. [1993]: «Le ralentissement et le rêve», N ouveaux Actes Sémiotiques 26-27.

FONTANILLE, J. et C. ZILBERBERg [1998]: T ension et Signification, H ayen, $M$ ardaga, Philosophie et langage.

GÉN IN ASCA, J. [1984] : «Leregard esthétique», Actessémi otiques-D ocuments VI, 58.

GREIM AS, A.J. [1984]: «Sémiotique figurative et sémiotique plastique», Actes sémiotiques-D ocuments VI, 60;

[1987]: D el'imperfection, Périgueux, Fanlac.

Greimas, A.J. et J. COURTÉS [1979 et 1986]: Sémiotique. Dictionnaire raisonné de la théorie du langage, Paris, $\mathrm{H}$ achette.

Greimas, A.J. et J. Fontan ILle [1991]: Sémiotique des passions, Paris, Seuil.

KEANE, T. [1991]: «Figurativité et perception», N ouveaux Actes Sémiotiques 17

M ALDIN EY, H . [1973] : Regard, parole, espace, Lausanne, Éd. L'Âged'H omme. Merleau-Ponty, M. [1945]: Phénoménologie de la perception, Paris, Gallimard:

[1964]: L'C Eil et l'Esprit, Paris, Gallimard.

M ESCH O N NIC, H . [1987] : «Soulages, le rythme et la lumière», Catalogue Soulages, Lyon, 73-85.

O UELLET , P. [1992] : «Signification et sensation », N ouveauxActesSémiotiques 20.

RAGON, M.[1990]: Les Ateliers de Soulages, Paris, Albin M ichel.

REN OUE, M . [à paraître] : Sémi otique et perception esthétique. Les vitraux et la lumière de Pierre Soulages à Sainte F oy de C onques, T oulouse, EU S;

[1996]: «Analyse sémiotique de la perception d'un objet du monde naturel », N ouveaux Actes Sémi otiques 48;

[1997]: «La couleur : un paradigmequi sejouedela rationalité?», Actes du colloqued'Albi 1996: Logiquesdeslangages, T oulouse, P.U .M ., 151-174. SCH APIRO, M . [1969] : «O n some problems in the Semiotics of Visual art: fields and vehicle in image-signs», dans Sémiotica I, 3, 223-242.

SCH ULZ, M . et C. Vogel [1995]: "La praxis énonciative», N ouveaux Actes Sémiotiques 41-42.

SJ ö LIN , J.-G . [1996] : «L'imagevisuelle- signification matérielle, plastique et iconique», Visio 1, 2, 43-48

TAN IZAKI, ]. [1933] : L'Éloge del'ombre, Paris, Publications orientalistes de France, 1993

ZILBERBERG, C. [1992]: «D éfense et illustration de l'intensité», dans]. Fontanille, La Q uantité et ses modulations qualitatives, Limoges, PU LIM ;

[1992] : «Présence de W ölfflin », N ouveaux Actes Sémi otiques 23-24. 